\title{
Systematic Process Synthesis and Design Methods for Cost Effective Waste Minimization
}

\author{
Final Report \\ Grant DOE-DE-FG02-85ER13396 \\ L. T. Biegler, I. E. Grossmann and AmWsterbefol
Chemical Engineering Departient
Carnegie Mellon University
Pitsburgh, PA 15213
}

This report focuses on research done over the past four years under the grant with the above title. In addition, the report also includes a brief summary of work done before 1994 under grant DOE-DE-FG02-85ER13396. Finally a complete list of publications that acknowledge support from this grant is listed at the end.

\section{Research from 1994-1998}

This project develops a novel integrated approach for process synthesis and design that addresses recent environmental challenges. Specifically, these synthesis approaches will provide rigorous trade-offs among raw material and energy costs, capital investment and waste treatment. To address the problem of waste minimization at the design stage, major issues include:

- Economic design of continuous and batch processes that minimize conversion of raw materials to waste byproducts.

- Synthesis of batch and continuous separation sequences to isolate, redirect and recycle byproducts.

- Integrated synthesis and design with environmental concerns, also dealing with process uncertainty.

Recent work on the first topic has been devoted to integrating dynamic operating conditions at the stage where the batch plant is designed and scheduling policies are considered. At this level there is tremendous scope for improving the profitability of the process as well as its efficiency in converting raw material to desired product. This work is related to and builds on previous work in developing superior reactor designs for continuous processes through dynamic optimization strategies. In both cases, by integrating various a:pects of design and operation, we see the greatest impact for process improvement, both from an environmental and an economic perspective.

The second topic considers the recovery and reuse of small amounts of byproducts, which can have a significant impact on waste treatment costs. Performing this task requires more exacting designs for systems with nonideal mixtures, such as the generation of flexible solvent recovery systems. A key component to this work is the generation and evaluation of complex column sequences. Such designs are large and contain severe nonlinearities. As a result, accurate but inexpensive shortcut ; nodels have been developed through collocation techniques.

Finally the third task considers the development of flexible designs that need to be tolerant to uncertainties related to process conditions and waste treatment requirements. In this 


\section{DISCLAIMER}

This report was prepared as an account of work sponsored by an agency of the United States Government. Neither the United States Government nor any agency thereof, nor any of their employees, make any warranty, express or implied, or assumes any legal liability or responsibility for the accuracy, completeness, or usefulness of any information, apparatus, product, or process disclosed, or represents that its use would not infringe privately owned rights. Reference herein to any specific commercial product, process, or service by trade name, trademark, manufacturer, or otherwise does not necessarily constitute or imply its endorsement, recommendation, or favoring by the United States Government or any agency thereof. The views and opinions of authors expressed herein do not necessarily state or reflect those of the United States Government or any agency thereof. 


\section{DISCLAIMER}

Portions of this document may be illegible in electronic image products. Images are produced from the best available original document. 
approach, design under uncertainty is considered through multiperiod strategies that deal with both continuous and discrete variables in an MNNP formulation. The resulting problem is large and expensive to solve, and as a result, a novel solution procedure has been developed that allows for efficient solution of these problems.

\section{Differential-Algebraic (DAE) Models for Batch Processing}

\section{Students: $\quad$ Tarun Bhatia, (Ph.D. completed April, 1998)}

An important task for process optimization is the integration of multiple objectives, tasks and subsystems for a process. For design, these include trade-offs with the efficiency of raw material conversion, capital cost and energy consumption. In addition to profitability, performance interactions should be considered that include controllability, safety and tolerance to uncertainties, both with the process model and process disturbances. Finally, in process planning, there are interactions with other processes and trade-offs in considering demands and capacities as well as expansions and long range outlooks. Process optimization formulations naturally and directly handle interactions, multiple conditions and trade-offs in an unambiguous, quantitative manner. On the other hand, the resulting formulations may lead to more difficult problems to solve. Therefore it is very important to determine:

a) which large-scale algorithms, software and hardware are available

b) what are the best problem formulations

c) what further algorithmic development is needed.

Over the past decade a number of optimization formulations have been developed for integration of batch process design and scheduling (Birewar and Grossmann, 1989; Voudouris and Grossmann, 1993) and design and dynamic performance (Logsdon and Biegler, 1993). We have considered the design, operation and scheduling of a batch process. In particular, we are interested in results from nonintegrated design and optimization formulations and the impact of integrated formulations. By combining these problem aspects, we hope for synergies that lead to shorter processing times, shorter planning horizons and fewer, higher quality batches. Two important aspects to this case study are the use of simultaneous dynamic optimization and a simplified closed-form scheduling formulation.

For the simultaneous dynamic optimization we discretize the differential equations (DAEs) and state and control profiles to form a large-scale optimization problem. This approach handles profile constraints and incorporates equipment design variables directly. The resulting formulation works well even for unstable systems and the DAE model is solved only once during the optimization. The closed form scheduling formulation is adapted from Birewar and Grossmann (1989) and deals with the sequencing of tasks, products and equipment using simplified Unlimited Intermediate Storage (UIS) and Zero Wait (ZW) transfer policies. In both cases, a nonlinear program is formulated and the solution directly yields a minimum cycle time operating schedule for multiple products and stages. The detailed integrated formulation is given in Bhatia and Biegler (1996a).

To demonstrate the potential of this approach, consider the process example given in Figure 1 , consisting of a reactor, heat exchanger, centrifugal separator and batch column. We consider the sequencing of three products $(\mathrm{P})$ of varying purity and with possible manipulations of the temperature profile in the reactor and the reflux profile in the column. We term the optimal dynamic profile cases $\mathrm{Rl}$ and $\mathrm{Cl}$ for the reactor and column, 
respectively. The best constant profile cases are termed $\mathrm{R} 0$ and $\mathrm{C} 0$. We also consider three profit maximization cases. First, we consider the sequential design where designs, dynamics and scheduling are optimized in sequence. The intermediate case deals with the final time states fixed by a unit optimization and then solution of a simultaneous design and scheduling. Finally, we consider a fully simultaneous approach with optimized final states. The optimal dynamic and constant profiles can be found in Bhatia and Biegler (1996a). The results of this case study are summarized in Figure 2 for the ZW case. Note that significant improvements result from the integrated formulation. Here we see that there is improvement due to a variable reflux ratio (from $\mathrm{C} 0$ to $\mathrm{C} 1$ ) but the greatest improvements are due to the integration of the design, operation and scheduling steps. The reason for this is apparent from the production schedule. The sequential solution still has long cycle times and long slack times in the $\mathrm{ZW}$ schedule. The intermediate solution reduces both of these considerably and the fully simultaneous approach eliminates the slack times altogether and creates a schedule with the shortest cycle time. This allows for much greater equipment utilization and significantly improves the profit.

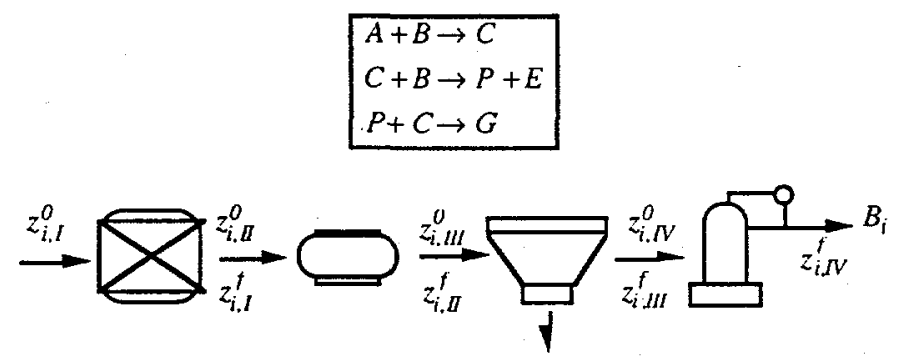

Fig. 1 Example of batch process

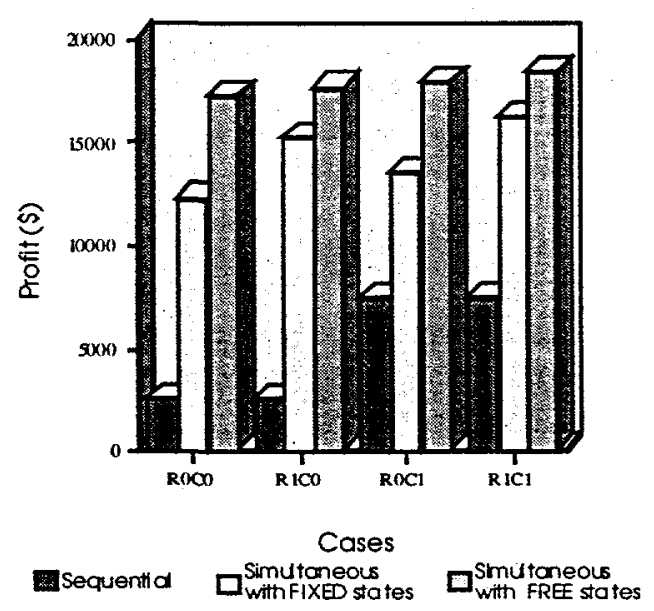

Figure 2: Results of Case Study for the $\mathrm{ZW}$ case

These results were obtained with the assumption of perfect operating and design models. However, uncertainty in these models can easily offset some of the gains accomplished through integrated formulations. These can arise from an imperfect model and model parameters, process disturbances and uncertain inputs, and from variations in demands and supplies in planning. Nevertheless, treating uncertainty can also be handled through optimization formulations. Whether the uncertainty exists in the model or through disturbances, these are frequently not known in advance. To deal with realistic problem formulations we can consider a robust (Here and Now or RI) formulation which ignores 
variations in the controls or a perfect information (Wait and See or PI) formulation which assumes $\theta$ is known and cannot be implemented. Clearly, the latter formulation leads to a better objective and we can define an expected value of perfect information. An implementable intermediate strategy uses feedback correction (FC) whereby the control profiles are determined through variations in the state variables, i.e.: $u_{i}=u_{n o m}+k\left(y_{i}-\right.$ Ynom). Here we can show that the feedback correction strategy is bounded by the PI and RI strategies and can recover some of the EVPI. In Bhatia and Biegler (1996b) multiperiod formulations were solved for the above batch problem with uncertainties in the rate constant and relative volatility. For the $\mathrm{ZW}$ case the feedback correction strategy recovers about $90 \%$ of the EVPI.

Finally, the challenge in considering process uncertainty lies in the inexpensive solution of multiperiod optimization problems. Varvarezos et al. (1992) developed an efficient decomposition strategy that was linear in the number of periods and was easily parallelized. As part of this work, Bhatia and Biegler (1998) developed an Interior Point method for multiperiod problems which helps preserve problem structure structure and speed convergence. This work builds on related work developed in our group for interior point methods for large-scale nonlinear programming. For multiperiod problems we developed and efficient decomposition strategies that is based on the bordered block diagonal structure of the KKT system. Exploiting this structure leads to a decomposition strategies that is linear in number of periods and also each period can be solved in parallel. Moreover, with enough processors, the cost of a multiperiod design could be that of a single optimization. In numerical comparisons on large dynamic optimization problems, Bhatia and Biegler (1998) showed that this approach can be orders of magnitude faster than general purpose optimization strategies.

\section{Synthesis of flexible solvent recovery processes}

\section{Student: Kenneth Tyner (PhD student started August, 1994)}

This part of the project deals with the design of flexible separation systems. As part of this work, we had developed a collocation model for distillation colum for analysis and flexible design (Huss and Westerberg, 1994; 1996). We also examine the design of complete flexible separation processes and have defined his problem as follows:

Separate for recycle the solvents received from several processes operating at a single plant site. The flow and composition of the solvent mixture we receive varies with time (e.g., each of the processes may change its operating level or shut down at any time). We characterize this variability by enumerating a set of different feeds. For each feed we specify the component flowrates and the fraction of time we will receive that feed to process.

This problem consists of three tasks. The first is to carry out a continuous variable optimization of a separation process composed of several columns. The process must be capable of carrying out each of the enumerated separation tasks. Selected are the diameter, number of trays and feed tray location for each column. At issue is whether one can get such a computation to converge successfully.

The second task is to enumerate all the possible alternative flexible configurations. For small problems, in excess of several thousand alternatives will exist. 
The third task is to investigate how to carry out the search over the alternative configurations, remembering that each will likely require a very costly continuous variable optimization.

We illustrate these steps by considering the solvents acetone, chloroform and benzene $(A C B)$. Fig. 3 is a composition diagram showing five different feed compositions we might wish to process over the course of a year. Solvents selected for a process are generally very dissimilar in nature. As such, mixtures of them will display azeotropic and liquid/liquid behavior. The ACB phase diagram at one atmosphere displays a maximum azeotrope between acetone and chloroform and a curved "distillation boundary" running from that azeotrope to the benzene corner. There are many aspects to this synthesis problem that make it very difficult. We discuss each in turn. Fig. 4 shows a separation process capable of separating the first feed shown in Fig. 3 into pure component products. Typical of separating azeotropic mixtures, one finds the need for recycles. We have readily enumerated three different configurations to separate feed 1 in Figure 3. Each of the other feeds has several different separation schemes possible.

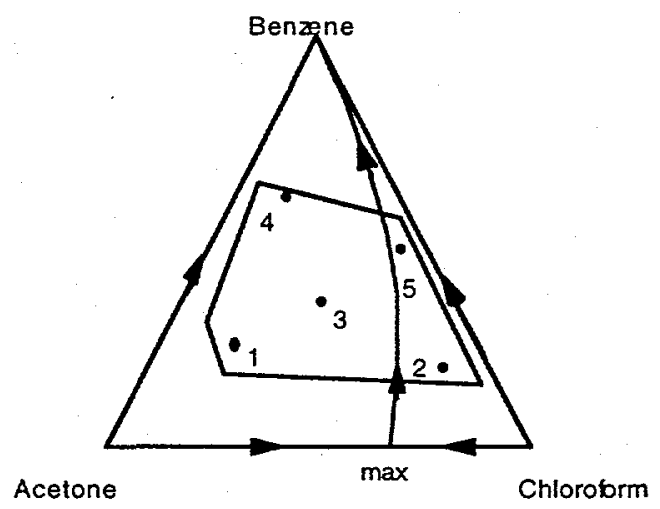

Fig. 3. Composition diagram for acetone, chloroform and benzene mixtures diplaying different feeds expected throughout a year of operation for a solvent recovery system

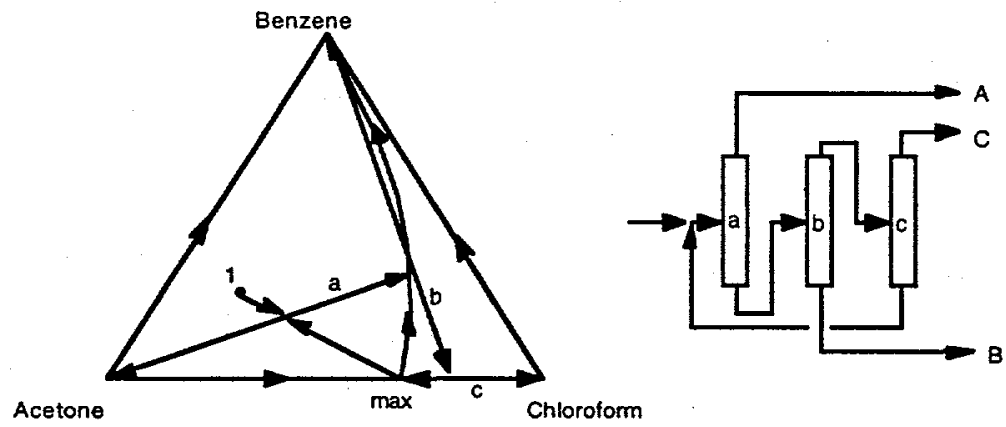

Fig 4. One of several separation schemes for processing feed 1

Suppose we have decided on a three column configuration we intend to use for separating each of the feeds. Since each of the feeds occurs at a distinct time, we can consider sharing equipment among these configurations. For example, suppose we have to process feed 1 during January. During February and March we have to process feed 2. Suppose both require three columns in their respective design configurations. There are many ways we could share equipment between these two configurations. For example, we could have six different columns. Or we could use column 'a' for feed 1 as column 'c' for feed 2 if it were designed appropriately (adequate number of trays, large enough diameter, designed for the pressure needed, etc). There are 120 ways to share these columns (e.g. $(\mathrm{a}, \mathrm{b}, \mathrm{c})(\mathrm{d}, \mathrm{e}, \mathrm{f})$; 
$(a, b, c)(a, e, f) ;(a, b, c)(d, a, f), \ldots$.$) For four feeds with each design configuration requiring$ four columns, the number of design alternatives is over 60,000 . With six columns and four feeds, the number grows to 1.7 million. The design of such processes could involve the saving of intermediates (e.g., the azeotropic mixture which is the distillate for column ' $c$ ' in Fig. 4) to be processed with a different feed at a different time. When we do, the number of alternatives explodes even more.

In the course of evaluating each of the columns in a design, we need to simulate a column. When mixtures to be separated display azeotropic and liquid/liquid behavior, they become markedly more difficult to simulate. Simulations using commercial distillation packages often fail. For example, consider column ' $b$ ' in Fig. 4. Its feed is in the distillation region to the left of the distillation boundary while its products are both in the distillation region to the right of the boundary. Unless one knows in which region the products will reside, it can be extremely difficult to make an initial guess for the simulation that can converge. Thus we should expect even when we propose a column as a part of a design, we may have difficulty in simulating it to discover how best to design it and estimate a cost for it. The column does not operate alone but rather within a system of columns having recycles of one or more of the intermediate and final products within it. Converging a simulation model for a system of columns, each difficult when simulating on its own, and having recycles among them is even more difficult.

Furthermore, for optimization, the number of trays in each of the column sections is an integer quantity which affects the simulation if we carry it out using tray by tray calculations. We have to add these to those variables we might use to select among the sharing and configuration alternatives we mentioned above. We have an extremely large mixed integer highly nonlinear optimization problem to solve which is highly prone to numerical failure while trying to solve. There are several approaches for solving problems of this type. One is to use methods like simulated annealing or genetic algorithms. These methods handle both discrete and continuous variables. However, these methods take thousands to tens of thousands of iterations. We choose not to afford such a large computational burden for our problem, which we have shown above is extremely large and difficult. Instead we use branch and bound methods to handle the search over the configuration decisions. We have identified computations that establish bounds on costs for such processes to make branch and bound methods effective. For example, we will compute a bound on the cost of a column in a configuration of columns by optimizing the configuration and having only the cost of that column as the objective function. A similar lower bound on utility cost for a column is also possible. These are not trivial computations to establish bounds, but, when they allow one to remove many of the designs possible from consideration, they should prove effective. We are also looking at using simplified models. The first is the use of collocation models for distillation columns. Collocation models convert the discrete variables characterizing the number of trays into continuous variables, a distinct advantage. These models also do not get larger for difficult separations as the model size reflects the order of the polynomials used to collocate and not the number of trays. Our collocation model is about the size of tray-by-tray models involving 18 trays for three component mixtures.

We are also developing simplified models of the composition space and its distillation boundaries. Such models are allowing us to develop very quickly material balance models for systems of columns having recycling intermediate and final products. When using these models, we are discovering even more design alternatives we overlooked previously. To search the enormous space of alternatives, we are developing a solution approach based on using so-called asynchronous teams (A-teams) of agents. Developed in the early 1980s at Carnegie Mellon in the Electrical and Computer Engineering department by Talukdar and his students, industry has used A-teams to solve several of their very large optimization 
problems effectively (circuit routing for VLSI, train rescheduling, and asynchronous traveling salesmen problems).

A-teams are teams of cooperating agents which attack the problem in parallel - rather like a colony of insects attacking a large systems problem such as building a home or finding and storing food. There are various agents doing different tasks. Each has a relatively simple and well-defined task. There are simulator agents, optimizer agents, heuristic configurers, destroyers, and so forth. They are autonomous because they decide when to run and on which problem to work. The only exchange of information among the agents is their sharing of their partial and complete solutions with each other. An example is to have several optimizers, one based on a genetic algorithm, another which is a hill climber, and a third which employs a branch and bound scheme to search over discrete decisions. The genetic algorithm will put solutions all over the solution space; thus it is very good at getting coverage of the space when optimizing. However, it is terribly slow to converge to a local optimum. The hill climbing algorithm can use good points generated by the genetic algorithm and march them to a local minimum. The branch and bound algorithm can take the better solutions found by any of the other algorithms and use them as upper bounds on the cost to trim the branches it has to search. The genetic algorithm can employ some of the solutions found by the others as parents from which to generate its next set of "children." Experience shows that A-teams often solve problems many times faster ( 100 times faster is not uncommon) and generally find better solutions than any of the algorithms used by any of the agents from which they are composed. We will be building our agents using the ASCEND modeling environment to carry out the simulation and continuous variable optimizations needed. We are currently completing this study and intend to create an Ateam having agents capable of synthesizing better solutions for separating a flexible solvent recovery system. This system will be demonstrated through the solution of several solvent recovery problems.

\section{MINLP Synthesis and Operation of Flexible Process Systems}

\section{Student: Ramesh Iyer (Ph.D. completed June 1997)}

The objective of this project has been to develop computational strategies for solving multiperiod MINLP synthesis models. We have considered the optimal design and operation of utility plants, as well as the optimal design and operation of oil platforms.

For the optimal multiperiod operation of utility systems it is assumed that an existing utility plant is given, as well as a number of time periods in which individual demands for steam and power can vary. The problem is to decide which equipment to turn on and off accounting for the changeover costs, which preclude that this problem be simpl; solved independently for each time period. The other complication that arises in this multiperiod MILP model is that because each time period involves $0-1$ variables, standard decomposition techniques such as Benders decomposition cannot be applied. We developed a strategy that is based on the idea of transforming the multiperiod problem into a shortest-path problem (Iyer and Grossmann, 1997a). The idea is to generate according to some criterion several MILP solutions at each time period, which are then linked accounting for the changeovers. This in effect is equivalent to synthesizing a network such as the one shown in Fig. 5 in which the optimal solution is found by a shortest path algorithm. Since it is in general not possit:e to generate the network once and for all, the procedure is iterative in nature involving an implicit enumeration scheme.

We applied this method to a 12 period problem of a utility plant with 2 boilers and 2 turbines and with the possibility of purchasing steam and electricity (Fig. 6). If the 
problem is solved as a single MILP it involves 204 0-1 variables, 721 continuous variables and 1053 constraints. This model could not be solved to optimality after 1 hour of CPU time with OSL. Instead, with the proposed method, the solution of 369 small MILP subproblems were required (17 0-1,53 continuous, 103 constraints). The computation requirement for solving the problem to optimality was 5 minutes. While the cost savings were modest $(3 \%)$ what is perhaps more significant is the nature of the solution. If the problem is solved independently for each time period, there are 25 predicted start-ups and shut-downs as seen in Fig. 7. With the simultaneous model there are only 5. Furthermore, while in the former there are 11 distinct period of operation, in the latter there are only 3 !

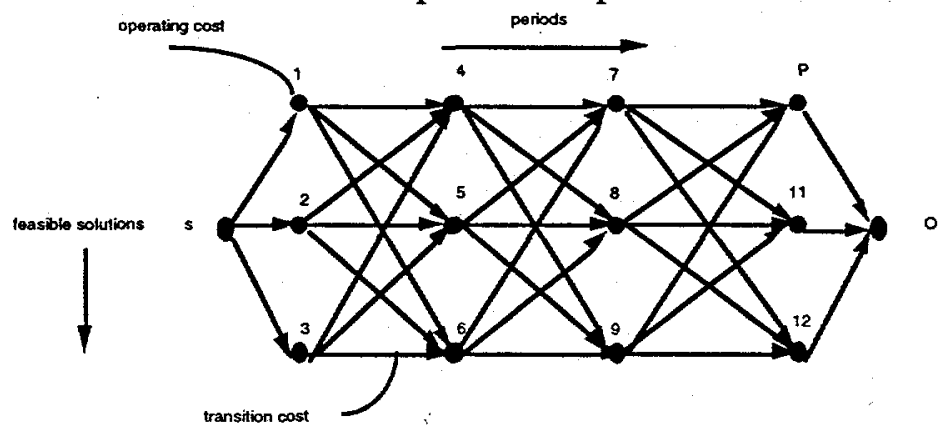

Fig. 5. Network representation of multiperiod operation

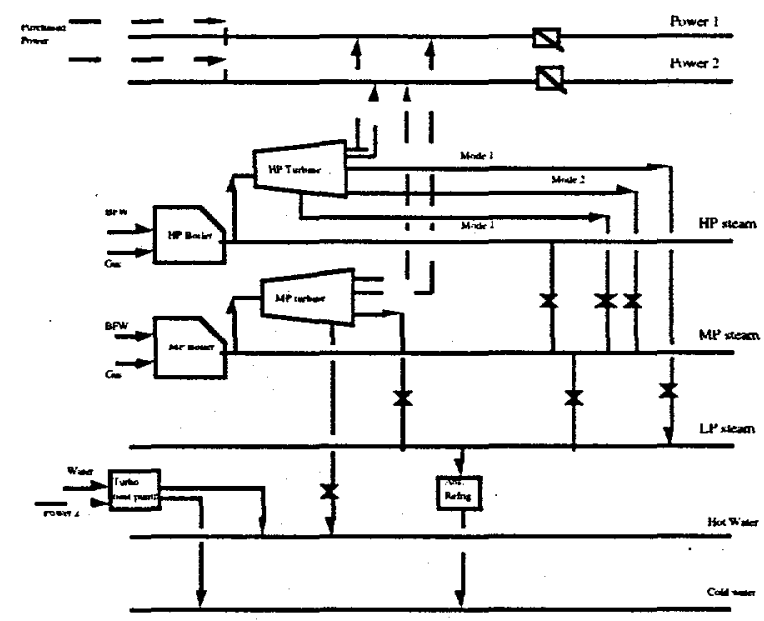

Fig. 6. Utility plant for small example.

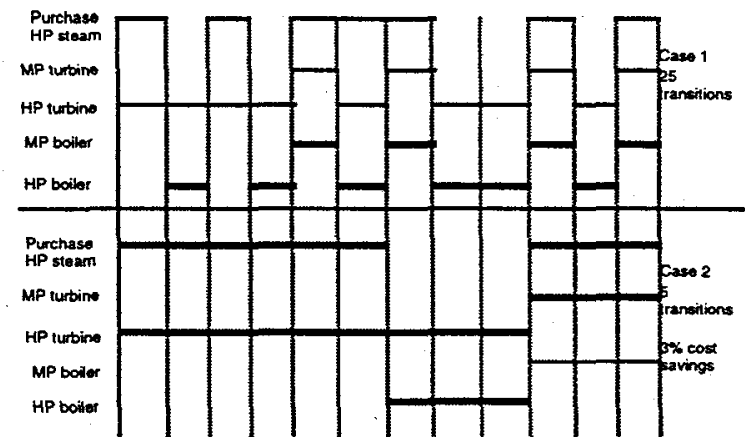

Fig. 7. Predicted shut-downs for independent optimizations (Case 1) and for simultaneous optimization (Case 2). 
We were able to extend the above problem to include both synthesis of the utility configuration as well as the operation over multiple time periods (Iyer and Grossmann, $1997 \mathrm{~b}$ ). In addition, we also considered as part of the problem that each unit must be shut down at least at one period in order to perform maintenance. The complication in this problem lies on the fact that it gives rise to a large scale MILP that is very difficult ad expensive to solve. Schemes such as Generalized Benders or aggregation methods proved to be unsuccessful. Therefore, we developed a special bilevel decomposition method that consists of two levels: design problem, and operational problem. The design problem corresponds to a relaxation that only involves $0-1$ design variables since the $0-1$ variables for each time period are eliminated, as well as the required lower bounds of activity for feasible operation. The operational problem arises with fixed 0-1 design variables, and gives rise to a problem almost identical to the one in the previous paragraph. The design problem predicts lower bounds while the operational problem predicts upper bounds. These two subproblems are solved successively by adding integer cuts and new design variable cuts to the design problem. We were able to demonstrate that this method is significantly faster than using a full space branch and bound enumeration. We also applied this method to a problem supplied to us by Mitsubishi Chemical in which we were able to predict large savings compared to a suboptimal solution obtained from the full space branch and bound method. It should be noted that we developed a similar bi-level decomposition solution approach for the multiperiod capacity planning and selection of process networks (Iyer and Grossmann, 1998a).

Finally, in cooperation with Mobil Oil, we also worked on the problem that deals with the optimal capacity selection of production and well platforms, and the scheduling of drilling of oil wells (see Fig. 8). This problem is also multiperiod in nature and has the added complexity that the description of the flow of the extracted crude is rather complex as it is nonlinear, and a function of the number and position of wells. We were able to develop a simplified MILP model by using piecewise linearizations. Since the multiperiod MILP model is quite large we developed a sequential hierarchical decomposition strategy that provide good suboptimal solutions (Iyer et al, 1998b). The basic idea relies on aggregating the time periods and the wells involved in a production field to define a higher level design problem that predicts an upper bound to the net present value. This model is used to select the wells that are to be exploited of the given time horizon. The problem is then disaggregated into selected wells and actual time periods, but solved in a rolling time fashion. This subproblem then defines the selection of platforms and their capacities. Finally, an optional subproblem is considered to "smooth" the production profiles (i.e. monotonic decrease) which then yields the final schedule. The quality of the solution can be assessed with the predicted upper bound. This decomposition strategy was applied to smaller test problems as well as to a real world problem. In the small problem, where we could also solve the MLP in the full space with CPLEX, the predicted optimum from our procedure was virtually identical. In the various problems the difference between the upper bound and the predicted solution was about $9 \%$. 


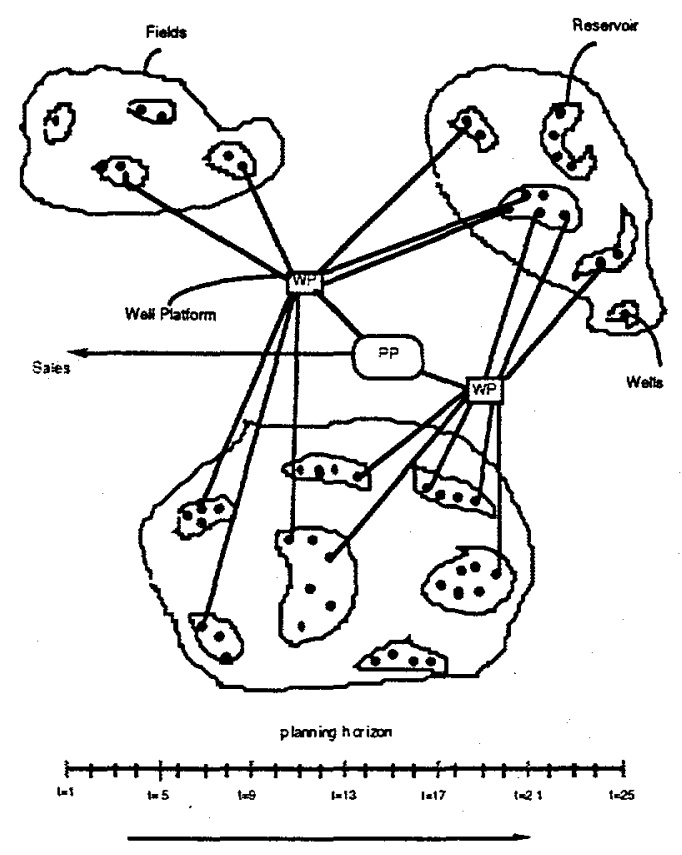

Fig. 8. Oilfield platforms and wells.

\section{REFERENCES}

Bhatia, T. K. and L. T. Biegler, "Dynamic optimization in the design and scheduling of multiproduct batch plants," I \& EC Research. 35, 7, 2234 (1996a)

Bhatia, T., and L. T. Biegler, "Dynamic Optimization in Planning Under Process Model Uncertainty," submitted for publication (1996b)

Bhatia, T. K. and L. T. Biegler, "Multiperiod design and planning with interior point methods," presented at Annual AIChE meeting, Los Angeles, CA (1997)

Birewar, D. and I. E. Grossmann, "Efficient algorithms for the scheduling of multiproduct batch plants," $I$ \& EC Research, 28, p.1333 (1989)

Huss, Robert S. and A. W. Westerberg, "Collocation Models For Distillation Design," Carnegie Mellon University, Engineering Design Research Center (1994)

Huss, R. S. and A.W. Westerberg, "Collocation Methods for Distillation Design 1: Model

Description and Testing," in press I\&EC Research, 1996

Iyer, R. and I.E. Grossmann, "Optimal Multiperiod Planning of Utility Systems", Computers and Chemical Engineering , 21, 787-800 (1997a).

Iyer, R. and I.E. Grossmann, "Synthesis and Operational Planning of Utility Systems for Multiperiod Operation", accepted for publication, Computers and Chemical Engineering (1997b).

Iyer, R. and I.E. Grossmann, "A Bilevel Decomposition Algorithm for Long Range Planning of Process Networks," Ind.Eng.Chem. Res. , 37, 474-481 (1998a).

Iyer, R. and I.E. Grossmann, "Optimal Planning and Scheduling of Offshore Oil Field Infrastructure Investment and Operations," Ind.Eng.Chem. Res., 37, 1380-1397 (1998b). 
Logsdon, J. S. and L. T. Biegler, "Accurate determination of optimal reflux policies for the maximum distillate problem in batch distillation," I \& EC Research, 32, 4, p. 692 (1993)

Varvarezos, D., L. T. Biegler and I. E. Grossmann, "Multiperiod design optimization with SQP decomposition," Comp. Chem. Engr.,18, 7, p. 579 (1992)

Voudouris, V. T., and I. E. Grossmann, "Optimal synthesis of multiproduct batch plants with cyclic scheduling and inventory consideration," I \& EC Research, 32, 2, p. 1962 (1993)

\section{Research Work Done Before 1994}

The main goal of this work, funded through DOE Grant DE-FG02-85ER13396, was the development of design methods that can explicitly address retrofit problems. The major challenge that lies in these problems is that they cannot be easily decomposed due to the interactions of the design with operational considerations. To address these problems, we developed models and computational techniques for the integration of design and operation, and several design methods which in fact are also relevant for grassroots problems. Our previous research work concentrated in the three following areas,

1. Efficient Algorithms for Large-Scale Optimization

2. Process Synthesis Strategies for Grassroots and Retrofit Designs

3. Analysis and Design for Process Operations

The first area has provided a fundamental aspect to the development of process design and redesign strategies. Most systematic approaches require the formulation and solution of large-scale nonlinear optimization problems that include discrete and/or continuous decision variables. We developed novel and efficient strategies for large-scale integer and nonlinear programming. These have also been specialized to parameter estimation problems that arise in process operations.

The second area has dealt more specifically with process design and redesign problems. Our research in this area has led to significant advances in the synthesis and redesign of energy management and separation systems. Here we have specifically considered the impact of highly nonideal thermodynamics in constructing economically viable separation sequences.

In addition, the operations aspects of process design have been considered in the following areas. First, very efficient integer programming algorithms were developed for optimizing the scheduling of operations in batch processes. Similarly, a metric was developed for assessing the uncertainties associated with discrete states as well as continuous process parameters that allow for the simultaneous consideration of flexibility and reliability for a given process. Finally, we developed efficient nonlinear programming algorithms that have led to faster algorithms for nonlinear model-based control: Here, stability properties of these control methods were also developed and refined.

Large-Scale Algorithms for Discrete and Continuous Variable Optimization Solution of specialized integer programming problems: A production planning problem for batch chemical processes frequently can be modelled as a symmetric travelling salesman problem (TSP), where each city represents a job and the distance between a pair of cities represents the changeover cost to setup the batch process from one job to the next. The solution of large TSP's is a very difficult combinatorial optimization problem and the largest such problems that could previously be solved exactly have no more than a few hundred cities (or batch jobs). Moreover since the volumes of production of such chemical 
products is often huge, a small improvement in solution quality could result in very large cost savings.

As part of the research supported by DOE, we have worked with Professor Joseph Pekny of Purdue and Dr. Donald Miller of Dupont on a new approach to finding exact solutions to random symmetric TSP's. Here we have devised a branch and bound algorithm, based on a relaxed two matching relaxation of the two matching constraints. This relaxation is solved by using a newly developed capacitated network flow algorithm to obtain surprisingly strong lower bounds. The largest problem that we have solved so far with this code is a 10,000 city random symmetric TSP, which took 11 minutes using a Sparc $1+$ work station. We are also implementing this method on the Nectar ring of computers at CMU, which will eventually connect all of the supercomputer centers in the country. Two papers are referenced that exposit the above procedures. The first (Miller et al, 1991a) contains computational results obtained with this algorithm for randomly-generated symmetric TSPs. The second paper (Miller et al, 1991b) specializes the methods in the first to find Hamiltonian cycles in a symmetric graph. Computational experience is reported on randomly generated problems with up to 30,000 nodes.

Efficient Algorithms for Large-Scale Nonlinear Programming: Process engineering optimization problems can be characterized by large, complex systems of algebraic and/or differential equations, but with relatively few degrees of freedom. To exploit this feature, we have developed a Range and Null space Decomposition (RND) for Successive Quadratic Programming (SQP). This has proven to be very successful for large-scale nonlinear programs and both "sparse" and "dense" versions have been implemented for this algorithm (Vasantharajan et al., 1990). As a result of this development nonlinear programming problems with as many as 8000 variables have been solved efficiently on workstation-class machines (Logsdon and Biegler, 1992). This approach has also been extended and specialized to two important problem classes in process engineering.

For the first problem class, our optimization/decomposition approach lends itself to tailoring to process simulation problems with specific model structure (e.g. banded systems, block bordered systems, etc.). In particular, the modified SQP algorithm has been tightly integrated to the Naphthali-Sandholm algorithm modelled in the SEPSIM simulator. The resulting distillation optimization requires less than $20 \%$ of the effort of a nested optimization (e.g. in sequential modular mode) and only twice the effort of the simulation itself. For the second problem class, a tailored SQP approach was developed for parameter estimation and data reconciliation. This strategy can be viewed as a constrained analog to the popular Levenberg-Marquardt method with quasi-Newton extensions and has also been specialized to deal with gross error detection and "errors in all variables" (EVM) problems. For gross error detection, a specialized maximum likelihood objective is used to track the "good" data while a much wider distribution is superimposed for the gross errors. This leads to reasonable, unbiased reconciliation of the data and a simple posterior test can be applied to detect and eliminate the gross errors (Tjoa and Biegler, 1992). For EVM problems an efficient algorithm has been developed that is up to two orders of magnitude faster than current approaches involving MINOS or SQP. Because of its decomposition properties we have also evaluated this algorithm on parallel architectures, and prototyped it on parallel machines at Argonne National Lab (Tjoa and Biegler, 1992a; 1992b). An invited presentation for the European Symposium on Computer Aided Process Engineering (Biegler, 1992) summarizes these tailored SQP strategies for the problems mentioned above.

\section{Process Synthesis Strategies for Grassroots and Retrofit Designs}


Optimal Design of Energy Management Systems: Our research work in this area concentrated first on the retrofit design of heat exchanger networks (Yee and Grossmann, 1991). The major result was the development of a screening strategy that was combined with the optimization of a novel superstructure for retrofit design in which the two dimensional layout of the network is accounted for. The role of the screening was to evaluate the economic potential of a retrofit design, and to suggest a bound on the number of additional units required. The optimization of the superstructure involved a large-scale MINLP which was solved with our MNNLP variant of the outer-approximation algorithm. A unique feature of this model is that it performs the simultaneous optimization of the energy recovery and investment cost, without relying on the common assumptions of fixed temperature approaches. For this reason the proposed method was able to predict a number of solutions that were not intuitively obvious.

Motivated by the improved results that we obtained in the retrofit problem through simultaneous optimization, we addressed grassroots design problems which in the past have been approached through sequential design strategies (e.g. targeting follwed by detailed synthesis). We developed a new superstructure representation that consists of a sequence of stages where each stream is successively split into matches and remixed. Using such a representation, the problem is formulated as an MINLP problem. An important feature in this model is that it can handle constraints such as no stream splits and forbidden matches, and does not rely on many of the restrictive assumptions of current methods (fixed HRAT, pinch partitioning, fixed EMAT's, etc). Furthermore, the model involves linear constraints as it relies on the assumption of isothermal mixing, which makes the model computationally efficient and robust. In contrast to pinch based design methods, the results have shown that the proposed model can synthesize networks that often require fewer number of units and that exhibit heat transfer across the pinch!. Furhermore, the proposed model tends to synthesize simple network structures. This model has been recently automated in the program SYNHEAT. This work was also expanded to perform simultaneous area and energy targeting, and simultaneous optimization and heat integration (Yee et al, 1990). An overview of this work was presented at the IEA Workshop on Process Integration in Gothenburg, Sweden in 1992.

Redesign of separation systems for azeotropic mixtures: The long term intent of this research is to understand how to redesign separation processes where the species display azeotropic behavior. Fluid-based processes to separate azeotropic mixtures typically include a combination of simple flashing, distillation, extractive distillation and liquid-liquid extraction. We have been investigating the following topics in this area: (1) understanding separation of a given mixture by one of the above processes; (2) selecting, if necessary, an extractive agent to aid in the separation (3) discovering separation piocesses which lead to desired products from the feed mixture.

For problem (1) we have developed a method which allows us to predict all "reachable products" for a three component, single feed, two product distillation column for azeotropic mixtures. It involves computing two trajectories - a "pinch point curve" and a residue curve and covers all products possiole from minimum to total reflux conditions. An interesting feature of the approach is that we can predict how far one can cross so-called "distillation boundaries." Earlier published work predicted products possible when using total reflux, but it missed products when tie topology of the solution space became more complex. Secondly, we have discovered a very useful and quite simple approach to identify azeotropic behavior in picking extractive agents for extractive distillation, using infinite dilution $\mathrm{K}$-values. One can examine the differences in infinite dilution $\mathrm{K}$-values for the different species in the proposed extractive agent to find useful agents. These ideas are sufficiently simple that we can readily teach them to our undergraduates. For problem (3) 
the literature contains little on the automatic generation of the structure of alternative separation processes to separate azeotropic mixtures and is instead directed to analyzing the performance of given separation schemes. We now have an approach to generate automatically a variety of separation schemes for complex mixtures and have applied the method to many different mixtures. For example, for methanol, acetone, n-pentane and water, several different schemes were quickly proposed, and later verified using ASPEN. One introduced no other species but used liquid/liquid extraction, extractive distillation and simple distillation to obtain $99.9+\%$ pure single component products. More details on our results of our research in these three areas in described in four papers in the publication list.

Finally, we have considered minimum reflux computations in these columns, particularly when the columns do not produce sharply split products. Previous methods to compute "pinch points" and to line these up into a subspace do not handle nonsharp products. We have developed a method to compute minimum reflux requirements for separating nonideal three component mixtures into products where both contain all three species in significant amounts. We also believe it can be extended for any single feed, two product column. The minimum reflux computations are being done using ASCEND, an environment we have developed for setting up, debugging and solving complex equation-based models.

\section{Analysis, Design and Control for Process Operations}

Design Optimization of Stochastic Flexibility: We investigated the development of a new stochasic metric that can account for uncertainties in continuous parameters (e.g. product demands), as well as in discrete states (e.g. equipment failure) in order to provide a unified measure for the flexibility and reliability of a process. We initially developed a stochastic flexibility index for continuous parameters in linear systems (Straub and Grossmann, 1990) in which we determined the probability over the feasible region given distribution functions of the uncertain parameters. We developed a method that is based on performing the integration of the joint distribution function through a quadrature formula, where the placement of the nodes is performed in a recursive fashion by exploiting the structure of the active sets so that the boundary of the feasible region is explicitly found. As for the treatment of the discrete states, we developed a highly effective bounding procedure that requires the evaluation of relatively few discrete states in order to obtain tight lower and upper bounds for the expected flexibility over all the states. We applied this method to a chemical complex, where the results showed that independent measures of flexibility and reliability can be somewhat misleading when interactions are not accounted for between the two. We also applied our methods to characterize the flexibility of multiproduct batch plants that exhibit uncertainties in the product demands and equipment failure (Straub and Grossmann, 1992b). We exploited the structure of the problem by showing that for the case of normal distributions for the demands, the multiple integral for the stochastic flexibility can be reduced to a one-dimensional integral. Also, to determine the optimal sizes of the equipment that maximize the stochastic flexibility, we were able to develop a nonlinear programming formulation which does not involve the integral since we can work in terms of the normal deviate. The significance of this work is that we can readily determine trade-off curves of cost versus flexibility which are especially useful in retrofit design problems.

In addition, we developed models and solution methods to evaluate and optimize the stochastic flexibility in systems described by nonlinear models (Straub and Grossmann, $1992 \mathrm{~b}$ ). We showed that the placement of quadrature points inside the feasible region can be posed as a nonlinear programming problem which can then be readily extended to the maximization of stochastic flexibility subject to a cost constraint. Since the size of this 
problem can be rather large we have developed a Benders based decomposition method. This method has been applied to the design of a plug flow reactor and to the design of a process flowsheet. The proposed method has shown to be computationally efficient, and it is a useful tool for generating trade-off curves of cost versus flexibility. Finally, we also made comparisons with the quadratic loss function by Taguchi which is concerned with minimizing deviations of target values. We were able to show that if one poses the design problem through a reward function, this problem captures trade-offs between the capability of handling parameter ranges (stochastic flexibility) and capability of delivering consistent performance (quadratic loss function).

Optimization Strategies for Nonlinear, Model Predictive Control: Algorithms related to Successive Quadratic Programming have recently become very useful for optimization-based control of nonlinear systems. Consequently, we are also developing optimization-based, nonlinear control strategies from a theoretical perspective. In particular, we have considered simpler linear systems to demonstrate the properties of Newton-type controllers and enhanced them with stabilization procedures. These enhancements also carry over to nonlinear control applications and include move suppression and filtering concepts as used in popular IMC and LQG (linear) controllers. This has led to more stable numerical algorithms in the design of moving horizon controllers (Oliveira and Biegler, 1991a).

An important element to enhancing these controllers has been the treatment of constraints in the control algorithm. Here the treatment of hard constraints with optimization-based methods can lead to loss of stability and degraded performance, particularly in processes with inverse responses. In this case, conventional constrained controllers (such as QDMC) invert (part of) the model over the output constrained portion. As a result processes with left half plane zeroes lead to unstable controllers. To treat this stability problem, we have developed and analyzed alternate methods that apply 1-norm and 2-norm penalty functions. Using concepts from nonlinear programming, we have provided an interesting interpretation of the sources of instabilities arising from hard constraints. Moreover, by using the 1-norm penalty function it can be shown that our constrained controller shares the same stability properties as the unconstrained one. From this result, a relaxation scheme is proposed that quantitatively provides for constraint relaxation in order to preserve controller stability. Finally, this approach uses multiplier information from quadratic programming algorithms and can be implemented on-line (Oliveira and Biegler, 1991b).

Finally, a prototype process control package has been developed within Mathematica that implements some of the above concepts for nonlinear model predictive control. Here state and parameter estimation for model predictive controllers will also be included in order to compensate for model mismatch and unmeasured disturbances. This approach takes advantage of powerful sensitivity and estimation tools and leads to an efficient and reliable process control strategy. In particular, this prototype system allows for the rapid development of additional applications.

\section{Publications}

Our research results under DOE Grant DE-FG02-85ER13396 have led to the following publications. All of these acknowledge full or partial support from the Department of Energy.

Bhatia, T. K. and L. T. Biegler, "Dynamic optimization in the design and scheduling of multiproduct batch plants," I \& EC Research. 35, 7, 2234 (1996a) 
Bhatia, T., and L. T. Biegler, "Dynamic Optimization in Planning Under Process Model Uncertainty," submitted for publication (1996b)

Bhatia, T. K. and L. T. Biegler, "Multiperiod design and planning with interior point methods," presented at Annual AIChE meeting, Los Angeles, CA (1997), submitted for publication.

Biegler, L.T., "Tailoring Optimization Algorithms to Process Applications" Proc. of European Symposium on Computer Aided Process Engineering-1, Elsinore, Denmark (1992)

Grossmann, I.E. "Mathematical Models for Heat Exchanger Network Synthesis", IEA Workshop on Process Integration in Gothenburg, Sweden (1992).

Grossmann, I.E., A.W. Westerberg and L.T. Biegler, "Retrofit Design of Processes", Proceedings of FOCAPO (eds. G.V. Reklaitis and H.D. Spriggs), pp. 403-442, Elsevier, Amsterdam (1987).

Huss, Robert S. and A. W. Westerberg, "Collocation Models For Distillation Design," Carnegie Mellon University, Engineering Design Research Center (1994)

Huss, R. S. and A.W. Westerberg, "Collocation Methods for Distillation Design 1: Model

Description and Testing," in press I\&EC Research, 1996

Iyer, R. and I.E. Grossmann, "Optimal Multiperiod Planning of Utility Systems", Computers and Chemical Engineering , 21, 787-800 (1997a).

Iyer, R. and I.E. Grossmann, "Synthesis and Operational Planning of Utility Systems for Multiperiod Operation", accepted for publication, Computers and Chemical Engineering (1997b).

Iyer, R. and I.E. Grossmann, "A Bilevel Decomposition Algorithm for Long Range Planning of Process Networks," Ind.Eng.Chem. Res. , 37, 474-481 (1998a).

Iyer, R. and I.E. Grossmann, "Optimal Planning and Scheduling of Offshore Oil Field Infrastructure Investment and Operations," Ind.Eng.Chem. Res., 37, 1380-1397 (1998b).

D. L. Miller, J. F. Pekny, and G. L. Thompson, "An Exact Two-Matching Based Branch and Bound Algorithm for the Symmetric Traveling Salesman Problem," Management Sciences Research Report, 566, Graduate School of Industrial Administration, Carnegie Mellon University, February, 1991.

D. L. Miller, J. F. Pekny, and G. L. Thompson, "An Exact Algorithm for Finding Undirected Hamiltonian Cycles Based on a Two-Matching Problem Relaxation," working paper, March, 1991.

Oliveira, N. and L.T. Biegler, "Extension of Multistep, Newton-type Control Methods for Nonlinear Process Control," Proc. American Control Conference, Boston, MA (1991a)

Oliveira, N. and L.T. Biegler, "Multistep Newton-type Methods for Nonlinear Process Control: Treatment of Constraints," presented at Annual AIChE Meeting, Los Angeles (1991b)

Straub, D.A. and I.E. Grossmann, "Integrated Stochastic Metric of Flexibility for Systems with Discrete State and Continuous Parameter Uncertainties", Comp. Chem. Eng., 14, 967 (1990).

Straub, D.A. and I.E. Grossmann, "Evaluation and Optimization of Stochastic Flexibility in Multiproduct Batch Plants", Comp. Chem. Eng., 16, 69 (1992a).

Straub, D.A. and I.E. Grossmann, "Design Optimization of Stochastic Flexibility", Comp. Chem. Eng., accepted for publication (1992b).

Tjoa, I-B, and L.T. Biegler, "Simultaneous Strategies for Data Reconciliation and Gross Error Detection of Nonlinear Systems," Computers and Chemical Engineering, 15, 10, p. 679 (1992) 
Tjoa, I-B, and L.T. Biegler, "A Reduced Successive Quadratic Programming Strategy for Errors-in-Variables Estimation," Computers and Chemical Engineering, 16, 6, p. 523 (1992)

Tjoa, I-B, and L.T. Biegler, "A Parallel Implementation for Data Regression with Implicit Models," Annals of Operations Research, to appear (1993)

Wahnschafft, O.M., T.P. Jurain and A.W. Westerberg, "SPLIT: a Separation Process Designer," Comput. Chem. Engng, 15, No. 8, 565-581 (1991).

Wahnschafft, O.M., P. Blania, J.P. LeRudulier, M. Thomas and A.W. Westerberg, "SPLIT: II. Automated Synthesis of Hybrid Separation Systems" European Symposium on Computer-Aided Process Engineering (ESCAPE-1), Elsinore, Denmark, 24-28 May (1992).

Wahnschafft, O.M., J.W. Koehler, E. Blass and A.W. Westerberg, "The Product Composition Regions of Single-Feed Azeotropic Distillation Columns," I\&EC Res, 31(10), 2345-2361 (1992).

Wahnschafft, O.M., J.P. LeRudulier and A.W. Westerberg, "A Problem Decomposition Approach for the Synthesis of Complex Separation Processes," paper 155c, Annual Meeting of the AIChE, Los Angeles, CA, Nov. (1991). Accepted (1993).

Wahnschafft, O.M., and A.W. Westerberg, "The Product Composition Regions of Azeotropic Distillation Columns: II. Separability in Two-Feed Columns and Entrainer Selection," paper 23e, AIChE Spring Meeting, Houston (1993), accepted for publication (1993).

Yee, T.F. and I.E. Grossmann, "A Screening and Optimization Approach for the Retrofit of Heat Exchanger Networks", I\&EC Res., 30, 146-162 (1991).

Yee, T.F., I.E. Grossmann and Z. Kravanja, "Simultaneous Optimization Models for Heat Integration. I. Area and Energy Targeting and Modeling of Multistream Exchangers", Comp. Chem. Eng., 14, 1151-1164 (1990).

Yee, T.F. and I.E. Grossmann, "Simultaneous Optimization Models for Heat Integration. II. Heat Exchanger Network Synthesis", Comp. Chem. Eng., 14, 1165-1184 (1990).

Yee, T.F., I.E. Grossmann and Z. Kravanja, "Simultaneous Optimization Models for Heat Integration. III. Process and Heat Exchanger Network Optimization", Comp. Chem. Eng., 14, 1185-1200 (1990). 